\title{
ARTICLE OPEN \\ Augmenting MNK1/2 activation by c-FMS proteolysis promotes osteoclastogenesis and arthritic bone erosion
}

Se Hwan Mun ${ }^{1}$, Seyeon Bae ${ }^{1}$, Steven Zeng ${ }^{1}$, Brian $\mathrm{Oh}^{1}$, Carmen Chai ${ }^{1}$, Matthew Jundong Kim (D) ${ }^{1}$, Haemin Kim ${ }^{1}$, George Kalliolias ${ }^{1}$, Chitra Lekha Dahia ${ }^{2,3,4}$, Younseo $\mathrm{Oh}^{5}$, Tae-Hwan Kim (iD) ${ }^{5,6}$, Jong Dae $\mathrm{Ji}^{7}$ and Kyung-Hyun Park-Min (D) ${ }^{1,4,8}$

Osteoclasts are bone-resorbing cells that play an essential role in homeostatic bone remodeling and pathological bone erosion. Macrophage colony stimulating factor (M-CSF) is abundant in rheumatoid arthritis (RA). However, the role of M-CSF in arthritic bone erosion is not completely understood. Here, we show that M-CSF can promote osteoclastogenesis by triggering the proteolysis of c-FMS, a receptor for M-CSF, leading to the generation of FMS intracellular domain (FICD) fragments. Increased levels of FICD fragments positively regulated osteoclastogenesis but had no effect on inflammatory responses. Moreover, myeloid cell-specific FICD expression in mice resulted in significantly increased osteoclast-mediated bone resorption in an inflammatory arthritis model. The FICD formed a complex with DAP5, and the FICD/DAP5 axis promoted osteoclast differentiation by activating the MNK1/2/EIF4E pathway and enhancing NFATc1 protein expression. Moreover, targeting the MNK1/2 pathway diminished arthritic bone erosion. These results identified a novel role of c-FMS proteolysis in osteoclastogenesis and the pathogenesis of arthritic bone erosion.

Bone Research (2021)9:45

; https://doi.org/10.1038/s41413-021-00162-0

\section{INTRODUCTION}

Rheumatoid arthritis (RA) is a chronic inflammatory and autoimmune disorder ${ }^{1}$. Bone erosion is one of the key clinical features of RA and is closely linked to impaired mobility in patients with $\mathrm{RA}^{2}$. However, the underlying mechanisms of arthritic bone erosion by osteoclasts have not been fully determined ${ }^{3}$. In addition to inflammatory cytokines such as tumor necrosis factor-alpha (TNFa), macrophage colony-stimulating factor (M-CSF) and its receptor c-FMS have also been implicated in the pathogenesis of RA and arthritic bone erosion ${ }^{4}$. In patients with RA, the level of M-CSF is increased in the serum and synovial fluid ${ }^{5}$, and inhibiting c-FMS activation attenuates the progression of joint inflammation and bone erosion in animal models of arthritis ${ }^{6}$. Despite the importance of M-CSF in the differentiation of myeloid cells ${ }^{7}$, very little is known about the molecular mechanism underlying the role of M-CSF/cFMS in arthritic bone erosion.

Ectodomain shedding is critical for the function of various membrane proteins. Many cell surface proteins, such as Notch, undergo proteolysis via regulated intracellular proteolysis (RIP) to generate functional small fragments of membrane-anchored proteins ${ }^{8}$. This process is mediated by a disintegrin and metalloprotease (ADAM) and $\gamma$-secretase. c-FMS also undergoes proteolysis by tumor necrosis a converting enzyme (TACE) and $\gamma$ secretase, generating small fragments that degrade once cells are exposed to an inflammatory stimulus ${ }^{9,10}$. c-FMS proteolysis is believed to cause its breakdown and termination of its functions ${ }^{10,11}$. Due to the importance of c-FMS in myeloid cells, the functions and downstream signaling pathways of c-FMS and its interacting ligands have been studied intensively. Despite this research, the role of c-FMS proteolysis remains largely unknown.

Osteoclasts are bone-resorbing cells derived from myeloid lineage cells that are responsible for arthritic bone erosion ${ }^{12-14}$. There are many cellular sensors and effector proteins that play roles in the generation and ultimate function of osteoclasts. Of those factors, M-CSF and receptor activator of NF-KB ligand (RANKL) are essential factors in the function and differentiation of monocytes and osteoclasts ${ }^{13-16}$. M-CSF signaling induces the expression of receptor activator of NF-KB (RANK), a receptor for RANKL, and RANKL then induces the expression of nuclear factor of activated T cells, cytoplasmic 1 (NFATc1), a master regulator of osteoclastogenesis, to initiate the osteoclast differentiation program $^{12}$.

Transcription factor networks involved in NFATc1 mRNA expression have been well characterized, but the regulatory mechanisms of NFATc1 protein expression remain unclear. mRNA translation is tightly controlled at multiple levels, and alterations in protein synthesis can lead to disease or cellular apoptosis ${ }^{17}$. The initiation of protein synthesis is a rate-limiting step. This step is facilitated by eukaryotic initiation factor (elF)4F, which binds to the $5^{\prime}$ cap, $m^{7} \mathrm{GTP}$, of mRNAs, to recruit the mRNA to the ribosome. elF4F is a multisubunit protein complex composed of elF4A, elF4E, and elF4G. elF4G is recruited to mRNA and forms the $43 \mathrm{~S}$ preinitiation complex, which consists of three protein family members: elF4GI (elF4G1), elF4GII, and death-associated protein 5

\footnotetext{
${ }^{1}$ Arthritis and Tissue Degeneration Program, David Z. Rosensweig Genomics Research Center, New York, NY, USA; ${ }^{2}$ Tissue Engineering, Regeneration and Repair, Hospital for Special Surgery, New York, NY, USA; ${ }^{3}$ Department of Cell and Developmental Biology, Weill Cornell Medical College, New York, NY, USA; ${ }^{4}$ BCMB allied program, Weill Cornell Graduate School of Medical Sciences, New York, NY, USA; ${ }^{5}$ Hanyang University Institute for Rheumatology Research, Seoul, Korea; ${ }^{6}$ Department of Rheumatology, Hanyang

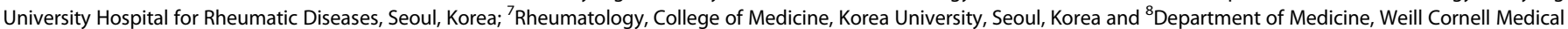
College, New York, NY, USA

Correspondence: Kyung-Hyun Park-Min (ParkminK@hss.edu)
}

Received: 15 October 2020 Revised: 16 April 2021 Accepted: 25 May 2021

Published online: 20 October 2021 


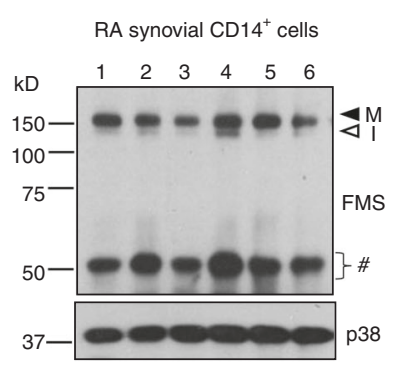

d

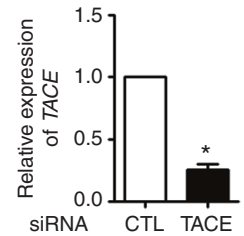

e

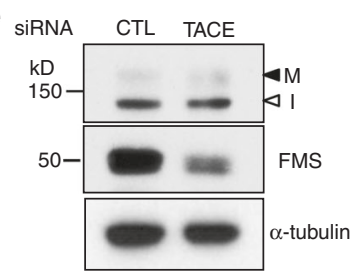

b

Healthy donors CD14 ${ }^{+}$cells

$\mathrm{M}-\mathrm{CSF} / \mathrm{h}-+++++++$

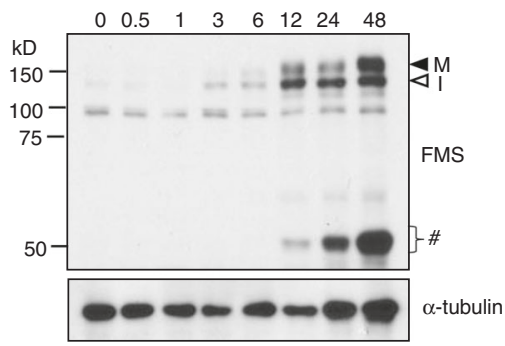

f

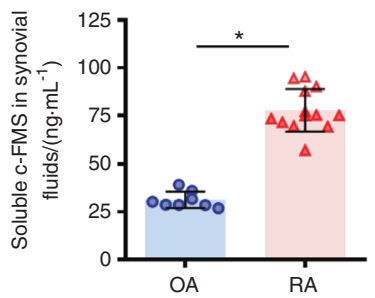

g

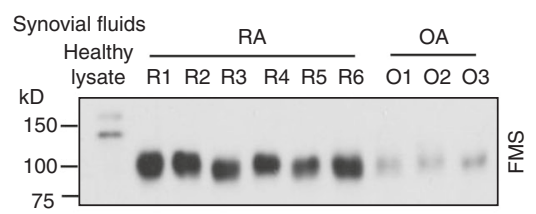

C $\mathrm{CD} 14^{+}$cells

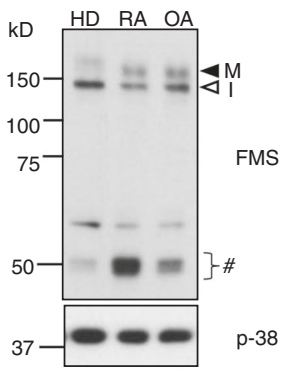

h
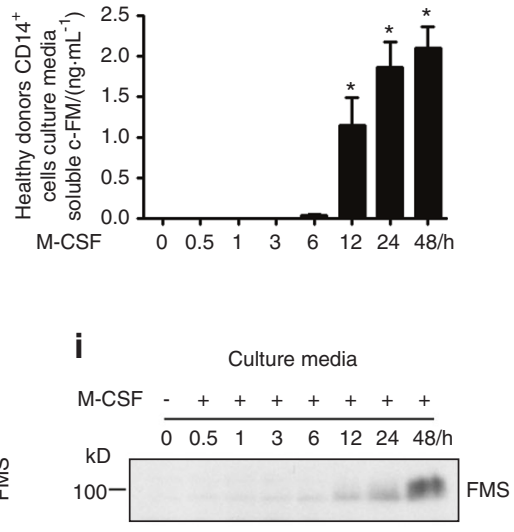

Fig. 1 The detection of small fragments of c-FMS and soluble c-FMS. a Immunoblot analysis of rheumatoid arthritis (RA) synovial CD14 ${ }^{+}$cells with antibodies against the C-terminus of c-FMS. $\mathbf{b}$ Human CD14 ${ }^{+}$cells from healthy donors were cultured with M-CSF for the indicated times. Immunoblot analysis of whole cell lysates with antibodies against the C-terminus of c-FMS. c Immunoblot analysis of whole cell lysates from $\mathrm{CD} 14^{+}$cells from healthy donors cultured with M-CSF for 1 day (HD) and synovial CD14 ${ }^{+}$cells from patients with RA or osteoarthritis (OA). d, e Human CD14 ${ }^{+}$cells were nucleofected with control (CTL) or TACE siRNAs and then cultured with M-CSF. d Knockdown (KD) efficiency. TACE mRNA was measured by qPCR and normalized to HPRT. e Immunoblot analysis of KD cells using an anti-c-FMS antibody. f, g Soluble c-FMS levels in synovial fluids from patients with rheumatoid arthritis (RA, $n=13)$ and osteoarthritis $(\mathrm{OA}, n=8)$ were measured by ELISA (f) and immunoblot analysis with antibodies against N-terminal c-FMS (g). $\mathbf{h}$, i Human CD14 ${ }^{+}$cells were cultured with M-CSF. Soluble c-FMS in the culture media was measured by ELISA (h) and immunoblot analysis with anti-c-FMS antibodies (i). All data are shown as the mean \pm SEM. ${ }^{*} P<0.05$ by unpaired $t$-test (d, f) or one-way ANOVA with a post hoc Tukey test (h). The data represent at least 3 independent donors. M; a mature c-FMS, I; an immature c-FMS, \#; small fragments.

(DAP5, also known as elF4G2). In contrast to the well-known function of elF4G1, the role of DAP5 in protein translation is controversial. A recent study showed that DAP5 could form inactive complexes and suppress protein $\operatorname{translation}^{18}$. Another study revealed that the DAP5 complex promoted alternative translation of specific subsets of mRNAs ${ }^{19}$. Furthermore, the full function of the DAP5 complex has not been defined, and the role of DAP5 in myeloid cells is unknown.

We showed that ligand engagement of c-FMS generated FMS intracellular domain (FICD) fragments in both human and murine macrophages via proteolysis. Here, we showed that increased FICD protein levels in arthritic synovial macrophages promoted osteoclast differentiation and arthritic bone erosion. Using gain-offunction and loss-of-function studies, we demonstrated that FICD fragments enhanced osteoclast differentiation and activity. Furthermore, myeloid-specific FICD transgenic mice exhibited an osteoporotic phenotype with increased osteoclasts and the promotion of arthritic bone erosion compared with those of control mice. This positive role of the FICD in osteoclasts was mediated by accelerating MAP kinase-interacting serine/threonine-protein kinase 1 (MNK1/2) activation and NFATc1 expression by binding to DAP5. Overall, our findings elucidate the molecular mechanisms of c-FMS proteolysis in osteoclasts and reveal how
c-FMS proteolysis accelerates RANKL-induced osteoclast differentiation and arthritic bone erosion. Our results also provide a strong basis for a new therapeutic target for pathological bone resorption in RA.

\section{RESULTS}

Synovial $\mathrm{CD}_{14}{ }^{+}$cells show a distinct c-FMS expression pattern M-CSF/c-FMS signaling is implicated in the pathogenesis of RA. Consistent with a previous report showing increased M-CSF expression in RA synovial fluids ${ }^{5}, M-C S F$ levels were significantly higher in RA synovial fluids than in osteoarthritis (OA) synovial fluids (Supplementary Fig. 1a). We also measured the expression of cell-associated c-FMS in synovial CD14 ${ }^{+}$cells from RA patients. A c-FMS antibody against the C-terminal region of the receptor detected mature, glycosylated c-FMS $(150 \mathrm{kD}, \mathrm{M})$ and immature, unglycosylated c-FMS (130 kD, I), as expected. Intriguingly, we also detected small fragments of $\sim 50 \mathrm{kD}$ in synovial CD14 ${ }^{+}$cells using anti-c-FMS antibodies (Fig. 1a). We next examined whether CD14 ${ }^{+}$ cells from healthy donors expressed small fragments. Immunoblot analysis of freshly isolated $\mathrm{CD}^{+} 4^{+}$cells showed low levels of mature and immature c-FMS, but small fragments were hardly detectable (Fig. 1b). After fresh $\mathrm{CD}_{14}^{+}$cells were cultured with 
M-CSF, the levels of mature and immature c-FMS and small fragments increased in a time-dependent manner (Fig. 1b). When we compared c-FMS expression between freshly isolated RA synovial $\mathrm{CD} 14^{+}$cells, M-CSF-cultured $\mathrm{CD}_{14}{ }^{+}$cells from healthy donors, and OA synovial CD14 ${ }^{+}$cells, we found higher levels of small fragments in RA synovial $\mathrm{CD}_{14}{ }^{+}$cells, while the levels of mature and immature c-FMS were comparable (Fig. 1c). To examine whether the observed $50-\mathrm{kD}$ bands originated from cFMS, mass spectrometry analysis was performed on the $50-\mathrm{kD}$ gel bands after immunoprecipitation with a c-FMS C-terminal antibody. Indeed, c-FMS was detected as the top ranked protein in the $50-\mathrm{kD}$ gel by mass spectrometry (Supplementary Fig. 1b). To corroborate our findings, we examined whether commercially available anti-c-FMS antibodies could detect the small fragments. The $50-\mathrm{kD}$ small fragments were detected by all antibodies against the C-terminal region of c-FMS (Supplementary Fig. 1c-e). However, these fragments were not detected by antibodies against the N-terminal region of c-FMS (Supplementary Fig. 1f, g). These results suggest that the $50-\mathrm{kD}$ bands contained C-terminal regions of c-FMS. We named these $50 \mathrm{kD}$ bands c-FMS intracellular domain (FICD) fragments.

Among peripheral blood mononuclear cells (PBMCs), only $\mathrm{CD}_{14}{ }^{+}$cells are able to differentiate into osteoclasts ${ }^{20}$. To examine whether FICD fragments were generated by c-FMS proteolysis, TACE expression was knocked down using short interfering RNAs (siRNAs) in freshly isolated CD14 ${ }^{+}$cells, and the cells were cultured with M-CSF. TACE was decreased by $75 \%$ by TACE siRNA compared with control siRNA (Fig. 1d). As a result, TACE knockdown diminished the generation of FICD fragments in response to M-CSF stimulation (Fig. 1e). Accordingly, treatment with the MMP inhibitor BB94 also suppressed the generation of FICD fragments (Supplementary Fig. 1h) and inhibited ectodomain shedding of c-FMS (Supplementary Fig. 1i). These results suggested that TACE cleavage was required for the generation of FICD fragments. To examine whether the increase in FICD fragments in RA synovial CD14 ${ }^{+}$cells correlated with the shedding of c-FMS, we measured the level of soluble c-FMS in RA and OA synovial fluids. Soluble c-FMS was detectable by ELISA and immunoblot analysis, and the level of soluble c-FMS was higher in RA synovial fluid than in OA synovial fluid (Fig. 1f). In addition, the soluble c-FMS in synovial fluids had a smaller molecular weight than full-length c-FMS (Fig. 1g), suggesting that c-FMS proteolysis may be active in the RA synovium. Accordingly, soluble c-FMS was not detected in freshly isolated $\mathrm{CD} 14^{+}$cells from healthy donors, but soluble c-FMS secretion in the media gradually increased during culture with M-CSF (Fig. 1h, i). However, other serological markers, including C-reactive protein (CRP), the erythrocyte sedimentation rate (ESR), and anti-cyclic citrullinated peptide (CCP) antibodies, were not associated with FICD expression in RA synovial $\mathrm{CD}_{14}{ }^{+}$cells (Supplementary Fig. 2a-c).

\section{M-CSF mediates the generation of FICD fragments}

Consistent with previous reports ${ }^{13,17}$, c-FMS proteolysis was initiated by TACE (Fig. 1). We reasoned that FICD generation followed a conventional RIP process through ADAM family proteins and $y$-secretase ${ }^{8}$. It has been shown that c-FMS has cleavage sites for $\gamma$-secretase in transmembrane regions ${ }^{10}$. Macrophages were treated with DAPT, a small molecule inhibitor of $y$-secretase $\mathrm{s}^{21}$. Then, the cells were lysed and fractionated into three cellular compartments: membrane, cytoplasmic, and nuclear. When we inhibited $\gamma$-secretase with DAPT, FICD remained in the membrane, and membrane-bound FICD accumulated (Fig. 2a). The membrane-bound form of the FICD had the highest molecular weight (mem), followed by the slightly smaller cytoplasmic FICD, which was denoted high molecular mass FICD (H-FICD). Both forms were larger than nuclear FICD, which was denoted L-FICD (low molecular mass FICD). We found that the cytosolic and nuclear FICD fragments were suppressed by DAPT (Fig. 2a). To further confirm the cellular localization of c-FMS and FICD fragments, we performed immunocytochemistry using an antibody against the C-terminal region of c-FMS in human macrophages, and signals were detected by fluorescence analysis and confocal microscopy. Consistent with the immunoblot analysis results, positive signals for c-FMS were detected in the membrane (mature form and mem), Golgi (immature form), cytoplasm (H-FICD), and nucleus (L-FICD) (Fig. 2b, Supplementary Fig. 3a). Since c-FMS signaling is required for FICD generation, we also examined whether c-FMS signaling contributed to the cellular localization of FICD fragments. Both M-CSF and IL-34, which are ligands for $\mathrm{C}-\mathrm{FMS}^{22}$, induced the generation of H-FICD and L-FICD and the cellular distribution of FICD fragments (Fig. 2c, Supplementary Fig. 3b, c). Macrophages were treated with imatinib mesylate, an inhibitor of c-FMS activity, or with a c-FMS blocking antibody, which suppressed not only FICD generation but also the levels of nuclear FICD fragments (Fig. 2d, e). Taken together, our results established that M-CSF/C-FMS signaling positively regulates the generation and cellular localization of FICD fragments in macrophages.

These results suggest that an additional protease may cleave $\mathrm{H}-\mathrm{FICD}$ to form L-FICD. To identify the protease(s) responsible for the cleavage of H-FICD in an unbiased manner, we screened 53 protease inhibitors using a protease library. The best hits associated with the inhibition of L-FICD generation were MDL28170 and PD150606 (two calpain inhibitors), along with MMP inhibitors and $\gamma$-secretase inhibitors. Calpain is a family of calcium-dependent cytosolic cysteine proteases expressed ubiquitously in mammals and many other organisms ${ }^{23}$, and calpaindependent cleavage contributes to the modulation of various cellular functions, such as apoptosis, proliferation and migration $^{24,25}$. Calpain has been implicated in osteoclastogenesis and migration 26,27 , although the exact mechanisms and targets of calpain are unknown. We found that inhibiting calpain suppressed the generation of the FICD in a dose-dependent manner (Supplementary Fig. 4a, b). The inhibition of calpain by MDL28170 did not interfere with the nuclear translocation of the FICD but instead shifted the enrichment of L-FICD to H-FICD in the nucleus, suggesting that calpain cleavage may occur in the nucleus (Fig. 2f). Since calpain is activated by calcium, we examined whether calcium signaling could compensate for M-CSF signaling to generate FICD fragments. In the absence of c-FMS signaling, calcium signaling was able to promote the cleavage of L-FICD to H-FICD in the nucleus, and MDL29170 reversed the effect of calcium signaling on L-FICD processing (Supplementary Fig. 4c). Consistent with the previous results, treatment with MDL28170 decreased not only L-FICD but also osteoclast differentiation (Supplementary Fig. 4d). To further investigate which form of calpain cleaves FICD in the nucleus, we used siRNAs to knock down Calpain 1, 5, and 6, which are expressed in macrophages. Calpain 1,5, and 6 were efficiently knocked down using siRNAs (Fig. 2g). Among them, calpain 1-knockdown (KD) cells were unable to process H-FICD to L-FICD, resulting in H-FICD accumulation in the nucleus in calpain 1-KD cells (Fig. 2h). Thus, our results reveal that calpain 1 plays a key role in the proteolysis of H-FICD to form L-FICD. To address the (patho)physiological importance of the FICD in inflammatory bone erosion, we examined the effect of MDL28170 on bone erosion in $\mathrm{K} / \mathrm{BxN}$ serum transfer-induced arthritis. K/BxN serum was administered intraperitoneally on day 0 and day 2, and then MDL28170 was administered after disease onset (Supplementary Fig. 4e). The severity of arthritis was assessed by clinical score and ankle joint thickness, which were attenuated by MDL28170 treatment (Supplementary Fig. 4f, g). Treatment with a calpain inhibitor decreased the number of osteoclasts and attenuated bone erosion in the $\mathrm{K} / \mathrm{BxN}$ serum-induced arthritis model (Supplementary Fig. 4h, i). Taken together, our results suggest that c-FMS 
a

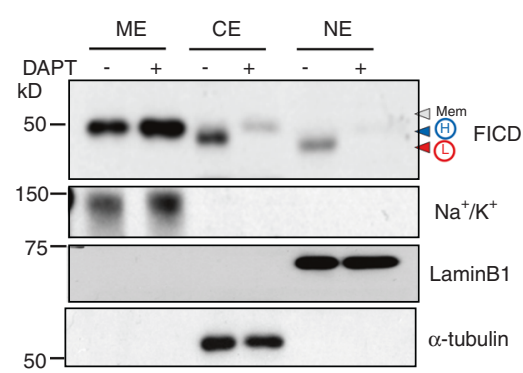

C

M-CSF - + + + + + - + + + +

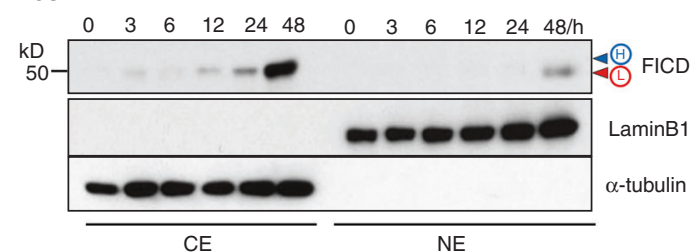

CE

f

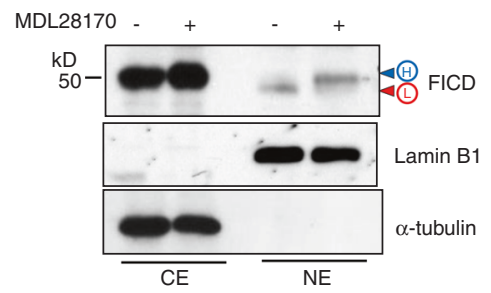

b

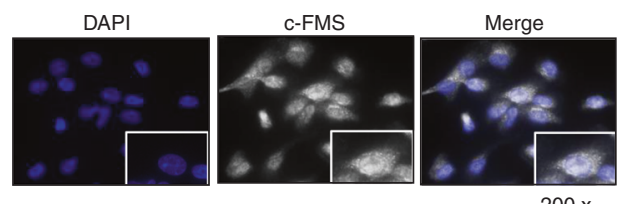

d

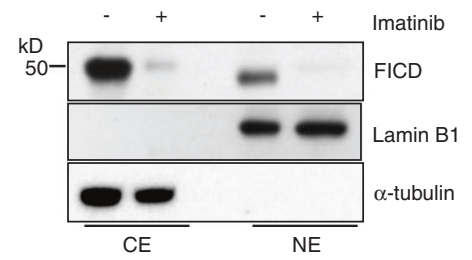

e

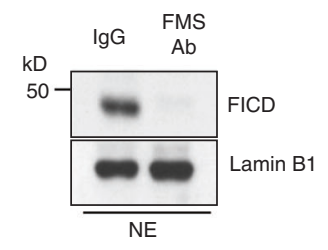

9

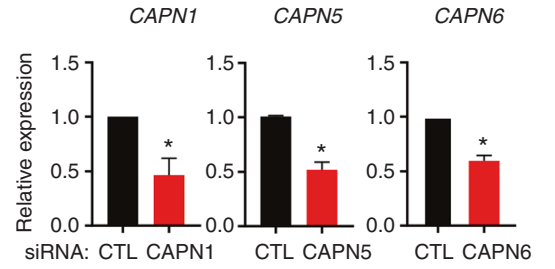

h

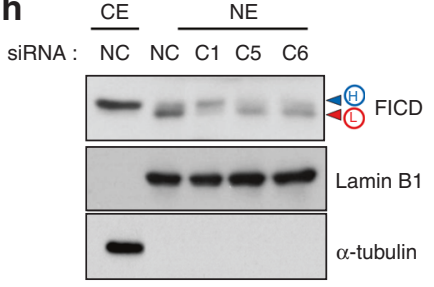

Fig. 2 Calpain 1 cleaves FICD fragments. a Human CD14 $4^{+}$cells were cultured with $\mathrm{M}-\mathrm{CSF}\left(20 \mathrm{ng} \cdot \mathrm{mL}^{-1}\right)$ for $8 \mathrm{~h}$ to induce early signals, and then DAPT $\left(10 \mu \mathrm{mol} \cdot \mathrm{L}^{-1}\right)$ was added. The cells were cultured for additional 2 days. Protein expression of c-FMS, the Na ${ }^{+} / \mathrm{K}^{+}$pump, Lamin B1, and $\alpha-$ tubulin were determined by immunoblot analysis. ME membrane extracts, CE cytoplasmic extracts, NE nuclear extracts. b Immunocytochemical analysis of DAPI and c-FMS. The right panel shows a merged image. Scale: $200 \times$. c Cells were starved for $3 \mathrm{~h}$ and then stimulated with M-CSF for the indicated times. d, e Cells were treated with imatinib $\left(0.3 \mu \mathrm{mol} \cdot \mathrm{L}^{-1}\right.$, d or a c-FMS-blocking antibody $\left(5 \mu \mathrm{g} \cdot \mathrm{mL}^{-1}\right.$, e prior to the addition of M-CSF. Protein expression of the FICD was measured by immunoblot analysis. Lamin B1 and $\alpha$-tubulin were used as controls for the nuclear and cytoplasmic fractions, respectively. f Human CD14 ${ }^{+}$cells were cultured with $\mathrm{M}^{-C S F}\left(20 \mathrm{ng} \cdot \mathrm{mL}^{-1}\right)$ for $8 \mathrm{~h}$ to induce early signals, and then MDL $28170\left(5 \mu \mathrm{mol} \cdot \mathrm{L}^{-1}\right)$ was added. The cells were cultured for additional 2 days. Immunoblot analysis with anti-c-FMS, Lamin B1, and $\alpha$-tubulin antibodies. g, h Calpain 1, 5, and 6 were knocked down with siRNAs. The cells were cultured with M-CSF for 12 h. $\mathbf{g}$ Efficiency of Calpain 1, 5, and 6 knockdown. $\mathbf{h}$ Immunoblot analysis of c-FMS, $\alpha$-tubulin, and Lamin B1. All data are shown as the mean \pm SEM. ${ }^{*} P<0.05$ by two-tailed, unpaired $t$-test $(\mathbf{g})$. Representative results from at least three independent experiments are shown.

proteolysis generates FICD though sequential proteolysis via TACE, $\gamma$-secretase, and calpain 1.

Blocking c-FMS proteolysis suppresses RANKL-induced osteoclast formation and activity

Given that FICD levels were increased in RA synovial CD14 ${ }^{+}$cells and that the administration of a calpain inhibitor suppressed both inflammation and bone erosion, we hypothesized that c-FMS proteolysis plays an important role in the functions of macrophages, including inflammatory responses and osteoclastogenesis. To test our hypothesis, we used noncleavable c-FMS mutants with mutated TACE cleavage sites (FMS ${ }^{\text {mut }}$, Fig. 3a) that could not produce FICD fragments ${ }^{10}$. 293 T cells that had no endogenous c-FMS expression were transduced with lentiviral particles encoding control, wild-type FMS $\left(\right.$ FMS $\left.^{\text {wt }}\right)$, or FMS ${ }^{\text {mut }}$. Cell surface expression of both FMS ${ }^{\text {wt }}$ and FMS ${ }^{\text {mut }}$ was determined by flow cytometry, and FMS ${ }^{\text {mut }}$ was resistant to TPA-induced TACEmediated shedding compared with FMS ${ }^{\text {wt }}$ (Supplementary Fig. 5a). Importantly, when the cells were stimulated with M-CSF, the activation of ERK, JNK, and p38 by FMS ${ }^{\text {mut }}$ was comparable to that of FMS ${ }^{\text {wt }}$ (Fig. 3b), suggesting that FMS ${ }^{\text {mut }}$ is a functional receptor. To minimize the effect of endogenous FICD, we used bone marrow-derived macrophages (BMDMs) from c-FMS inducible conditional haplodeficient mice (c-FMS ${ }^{f /+\Delta M \times 1}$ ) that were generated by crossing c-FMS floxed mice with $M x 1$ cre mice $^{28}$, which expressed low levels of endogenous FICD (Supplementary Fig. 5b). FMS haplodeficient BMDMs from $\mathrm{FMS}^{\mathrm{f} /+} \mathrm{M} \times 1 \mathrm{cre}$ mice were transduced with lentiviral particles encoding control, $\mathrm{FMS}^{\mathrm{wt}}$ or $\mathrm{FMS}^{\mathrm{mut}}$. As expected, FICD generation in both $\mathrm{FMS}^{\text {mut }}$. transduced FMS haplodeficient BMDMs and 293 T cells was diminished compared to that in FMS ${ }^{\text {wt- }}$ transduced cells (Supplementary Fig. 5c, d). To test the effect of c-FMS proteolysis on inflammatory responses, control, $\mathrm{FMS}^{\mathrm{wt}}$-, and $\mathrm{FMS}^{\mathrm{mut}}$-transduced cells were stimulated with LPS, a Toll-like receptor 4 (TLR4) agonist, and we measured the expression of proinflammatory cytokines such as TNFa and IL6. The mRNA expression of TNFa and IL6 was induced by LPS and was comparable among the groups (Fig. 3c). Both FMS $^{\text {wt }}$ and FMS ${ }^{\text {mut }}$ significantly increased the protein expression of TNFa and IL6 upon LPS stimulation (Fig. 3d). However, the levels of TNFa and IL6 were comparable between FMS ${ }^{\text {wt }}$ - and FMS ${ }^{\text {mut }}$-transduced cells (Fig. 3d). These results suggest that FICD fragments may have minimal effects on inflammation, while FMS ${ }^{\text {mut }}$ is functionally active and can promote inflammatory responses. Next, we examined the role of c-FMS proteolysis in osteoclastogenic responses to the TNF family cytokine RANKL. M-CSF signaling is a key regulator of osteoclast differentiation ${ }^{12}$. As expected, ectopic expression of $\mathrm{FMS}^{\mathrm{wt}}$ enhanced osteoclast differentiation and bone resorption compared with those of control cells (Fig. 3e). Strikingly, ectopic expression of FMS $^{\text {mut }}$ led to diminished osteoclast formation 
a

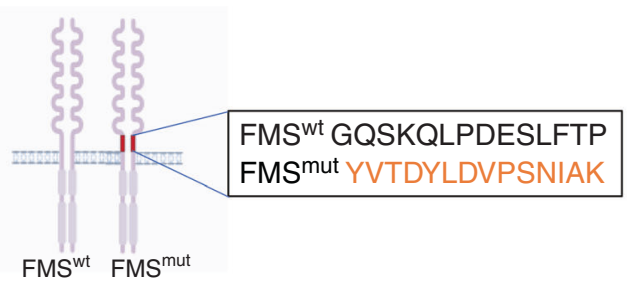

C
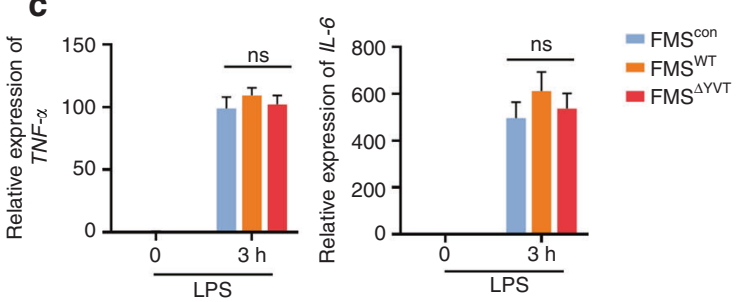

e
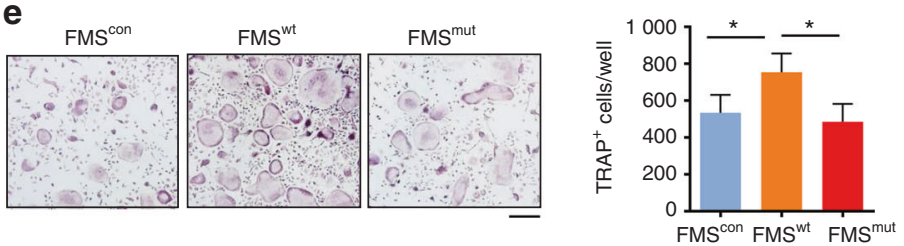

d
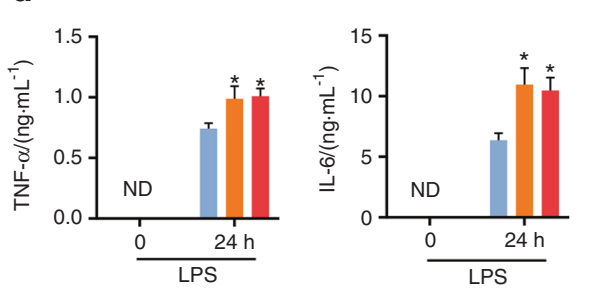

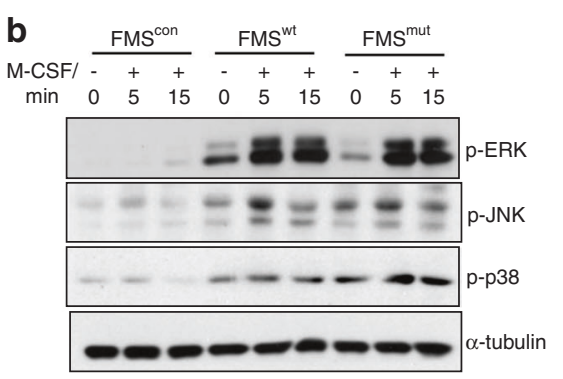

Fig. 3 c-FMS proteolysis positively regulates osteoclastogenesis. a Schematic showing mutations in the TACE cleavage sites of c-FMS. TACE cleavage sites of c-FMS ${ }^{10}$ were replaced by the addition of 14 amino acids from insulin receptor sequences (FMS ${ }^{\mathrm{mut}}$ ). b $293 \mathrm{~T}$ cells did not express c-FMS and were transduced with lentiviral particles encoding control, FMS ${ }^{\text {wt }}$ or FMS ${ }^{\text {mut }}$. The cells were then stimulated with M-CSF for the indicated times. Protein expression of phospho-ERK, phospho-JNK, phospho-p38, and $\alpha$-tubulin was determined by immunoblot analysis. c-f BMDMs from c-FMS ${ }^{\mathrm{f} /+}$ Mx1-Cre mice were transduced with lentivirus encoding control, wild-type FMS (FMS ${ }^{\mathrm{wt}}$ ), or the TACE-uncleavable mutant FMS (FMS ${ }^{\text {mut }}$ ). The transduced BMDMs were stimulated with LPS (10 ng. $\mathrm{mL}^{-1}$ ) for $3 \mathrm{~h}$ (c) and $24 \mathrm{~h}$ (d). c The mRNA expression of TNF $\alpha$ and IL6 was measured by q-PCR. d TNF $\alpha$ and IL6 protein levels in the culture media were measured by Luminex multiplex cytokine assays. e Osteoclastogenesis assay. The left panel shows representative images of TRAP-stained cells. The right panel shows the percentage of TRAPpositive multinuclear cells (MNCs: more than three nuclei) per control $(n=6)$. Black scale bar: $100 \mu \mathrm{m}$. f Resorption pit assay. Bone resorption activity analysis of FMS ${ }^{\mathrm{con}}$, FMS ${ }^{\mathrm{wt}}$, or FMS ${ }^{\mathrm{mut}}$ cells. The left panel shows representative images, and the right panel shows the percentage of the resorbed pit area per total area. Red scale bar: $200 \mu \mathrm{m}$. All data are shown as the mean \pm SEM. ns not significant, ND not detected. ${ }^{*} P<0.05$ by one-way ANOVA with a post hoc Tukey test (c-f). The data represent at least three experiments (b-d, $\mathbf{f})$.

relative to that of FMS ${ }^{\text {wt }}$-expressing cells (Fig. 3e), indicating that FMS $^{\text {mut }}$ could not efficiently promote osteoclastogenesis in the same manner as FMS ${ }^{\text {wt }}$. Concomitantly, the increased bone resorption activity of FMS ${ }^{\text {wt }}$-expressing cells was also diminished in FMS ${ }^{\text {mut }}$-expressing cells (Fig. 3f). Therefore, our results suggested that increased FICD levels in FMS ${ }^{\text {wt }}$-expressing cells contribute to osteoclast differentiation and activity but have no effect on inflammatory responses.

FICD knock-in mice exhibit an osteoporotic phonotype

To further delineate the role of the FICD in osteoclasts, a DDKtagged FICD was generated based on $\mathrm{N}$-terminal sequencing and predicted protease cleavage sites by the GPS-CCD program $^{29}$ (Supplementary Fig. 6). BMDMs were transduced with retroviral particles encoding DDK-tagged FICD. FICD protein expression was increased in FICD-transduced cells (Fig. 4a). Ectopic FICD expression enhanced RANKL-induced osteoclast differentiation and resorption compared with those of control cells (Fig. 4b, c), suggesting that constitutive FICD expression promotes osteoclastogenesis.

Increased FICD expression was observed in macrophages from RA patients (Fig. 1). To model the high expression of the FICD in vivo, we generated myeloid cell-specific conditional FICD knock-in mice (FICD ${ }^{K I / K I} \times$ Lyz2-cre ${ }^{\text {het }}$ mice) by crossing $\mathrm{FICD}^{K I / K I}$ mice with myeloid cell-specific LysM-driven CRE recombinase mice (FICDtg ${ }^{\mathrm{M}}$; Supplementary Fig. 7). FICD expression was measured by immunoblotting using anti-HA antibodies and was effectively increased in BMDMs (Fig. 4d). We examined whether the FICD regulates in vivo osteoclastogenesis. Micro-CT analysis showed that both FICDtg ${ }^{\mathrm{M}}$ male and female mice exhibited decreased bone mass, and the bone volume/tissue volume (BV/ TV) ratio and trabecular number (Tb.N) were significantly decreased compared with those of control mice (Fig. 4e, f, Supplementary Fig. 8a, b). Cortical thickness was comparable between control and FICDtg ${ }^{\mathrm{M}}$ mice, while cortical porosity was higher in FICDtg ${ }^{\mathrm{M}}$ mice (Fig. $4 \mathrm{~g}, \mathrm{~h}$ ). Histomorphometric analysis also showed that the number of osteoclasts, osteoclast surface area, and eroded surfaces were significantly higher in FICDtg ${ }^{\mathrm{M}}$ mice than in control LysM cre (WT) mice (Fig. 4i, j). Accordingly, serum CTX was higher in FICDtg ${ }^{\mathrm{M}}$ mice than in control mice (Fig. 4k). However, there were no differences in the mineral apposition rate (MAR) or bone formation rate (BFR) (Supplementary Fig. 8c-e). Overt phenotypic features, including body weight, spleen weight, and femur length, were not different between control and FICDtg ${ }^{\mathrm{M}}$ mice (Supplementary Fig. 8f-i), suggesting that FICD overexpression in myeloid cells did not affect the gross phenotype. In addition, we generated FMScKOFICDtg ${ }^{\mathrm{M}}$ mice by crossing $c-F M S^{f / f \Delta M \times 1}$ mice with FICDtg ${ }^{\mathrm{M}}$ mice. FICDtg ${ }^{\mathrm{M}}$ mice on a c-FMS-null background also exhibited diminished bone mass compared with control c-FMS-null mice (Supplementary Fig. 9a, b) 

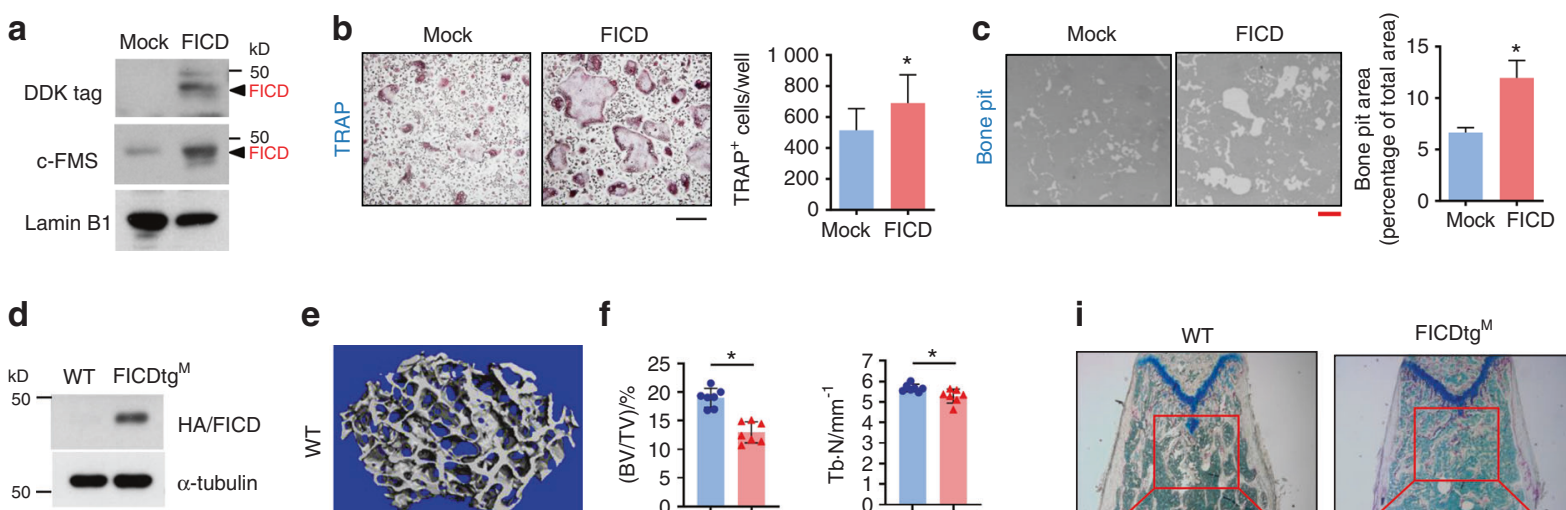

e
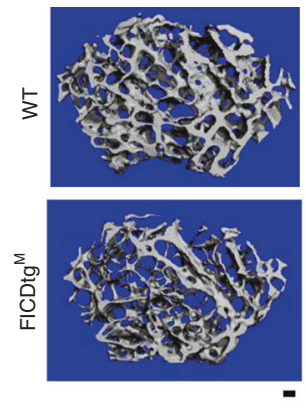

$f$

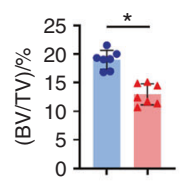

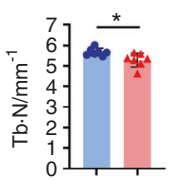
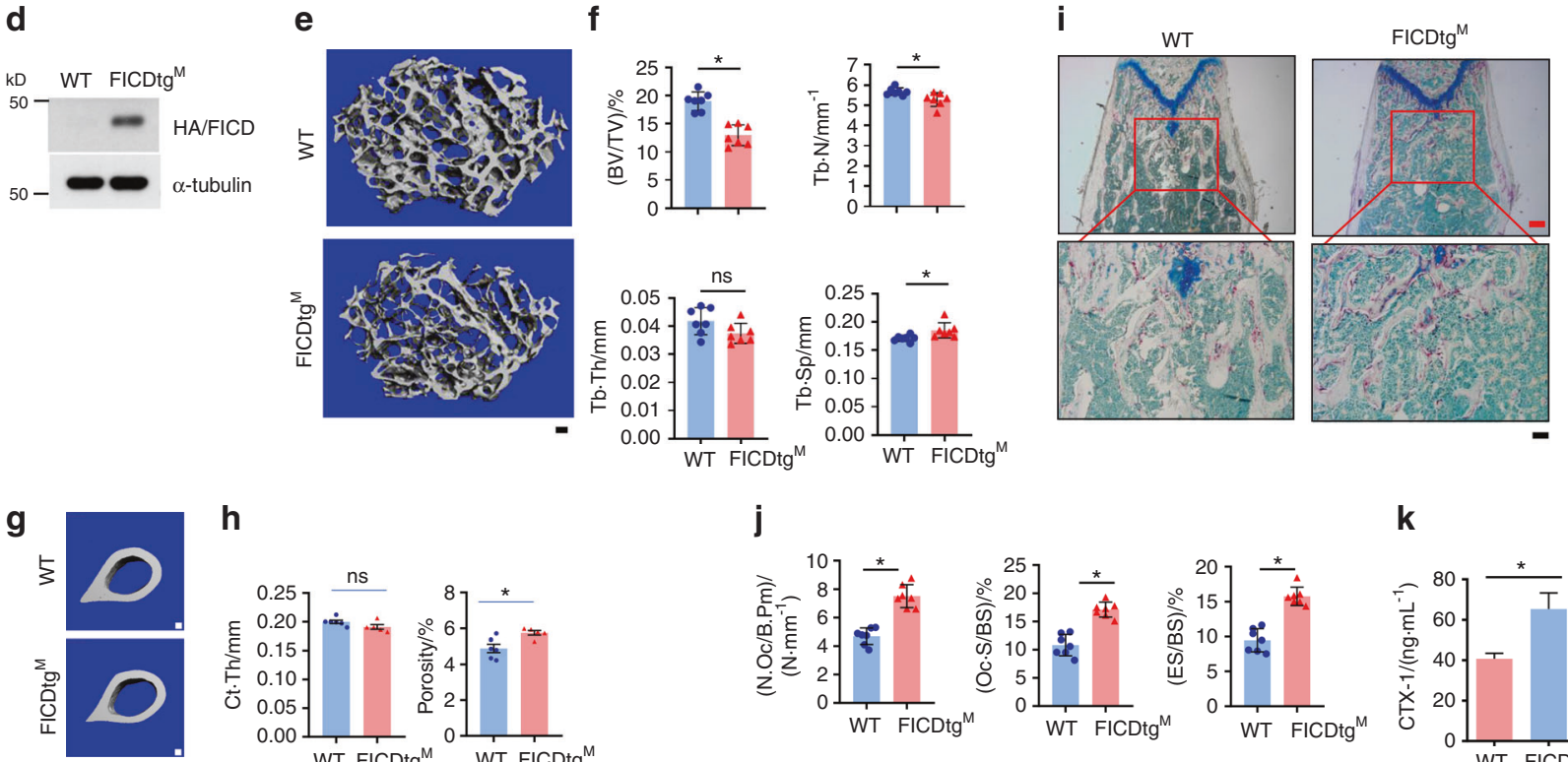

h

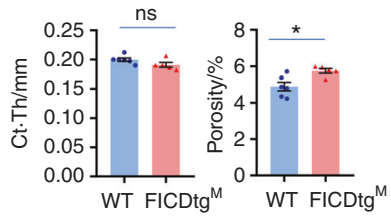

j

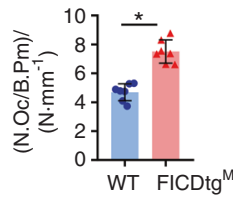

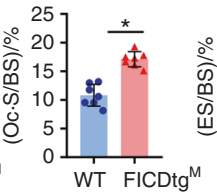

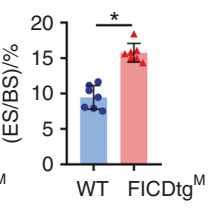

$\mathbf{k}$

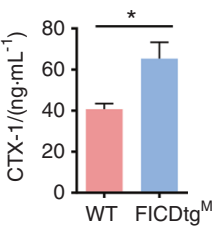

Fig. 4 FICDtg ${ }^{\mathrm{M}}$ mice exhibit an osteoporotic bone phenotype with increased osteoclast numbers. $\mathbf{a}, \mathbf{c}$ BMDMs were transduced with retroviral particles encoding either control or FICD. a The protein expression of DDK-tagged FICD was determined by immunoblot analysis. b Osteoclastogenesis assay. The left panel shows representative images of TRAP-stained cells $(n=4)$. c Bone resorption activity analysis. The left panel shows representative images, and the right panel shows the percentage of the resorbed pit area per total area ( $n=3$ ). $\mathbf{d}$ BMDMs from wild type (WT) and FICDtg ${ }^{\mathrm{M}}$ mice. Immunoblot analysis of the expression of HA-tagged FICD protein. e-h Micro-CT analysis of femurs from 12-week-old male wild-type (WT) and FICDtg ${ }^{M}$ mice $(n=7)$. Scale bar: $100 \mu \mathrm{m}$. e Representative images of distal femurs. f Bone parameters in distal femurs. Bone volume/tissue volume ratio (BV/TV), trabecular thickness (Tb.Th), trabecular number (Tb.N), and trabecular space (Tb.Sp) were determined by micro-CT analysis. g Representative images of cortical bone. $\mathbf{h}$ Cortical bone parameters. Cortical bone thickness (Ct.Th) and Cortical porosity. $\mathbf{i}$, j Histomorphometric analysis of the distal femurs of 12 -week-old male WT and FICDtg ${ }^{\mathrm{M}}$ mice $(n=6)$. i Representative image showing TRAP-positive, multinucleated osteoclasts (red). $\mathbf{j}$ The number of osteoclasts per bone surface (N.Oc/BS), osteoclast surface area per bone surface (Oc.S/BS), and eroded surface per bone surface (ES/BS). $\mathbf{k}$ CTX-1 (WT $=5$, FICDtg $\left.{ }^{\mathrm{M}}=8\right)$ in the sera of WT and FICDtg ${ }^{\mathrm{M}}$ mice. All data are shown as the mean \pm SEM. ns, not significant. ${ }^{*} P<0.05$ by two-tailed, unpaired $t$-test $(\mathbf{b}, \mathbf{c}, \mathbf{f}, \mathbf{h}, \mathbf{i})$.

and showed increased in vivo osteoclast activity (Supplementary Fig. 9c, d). Overall, our findings suggest that FICD expression in macrophages results in decreased bone mass by increasing osteoclast numbers under physiological conditions.

The FICD accelerates arthritis-induced bone erosion Given the high FICD levels in synovial CD14 ${ }^{+}$cells and its positive regulation of osteoclastogenesis without any effect on inflammatory responses, we hypothesized that the FICD may play a role in arthritic bone erosion. We first determined the effects of the FICD on inflammation and osteoclast differentiation in vitro. BMDMs from FICDtg ${ }^{\mathrm{M}}$ mice or WT mice were cultured with M-CSF and RANKL to form osteoclasts in vitro. Consistent with the in vivo data, FICDtg ${ }^{\mathrm{M}}$ cells showed significantly enhanced osteoclast differentiation and bone resorption activity relative to control cells (Fig. $5 \mathrm{a}, \mathrm{b}$ ). To examine the role of the FICD in inflammation, macrophages were stimulated with LPS $\left(10 \mathrm{ng} \cdot \mathrm{mL}^{-1}\right)$. The LPSinduced mRNA and protein expression of TNFa and IL6 were comparable between FICDtg ${ }^{\mathrm{M}}$ cells and control cells (Fig. 5c, d).

To address the importance of the FICD in osteoclast-mediated pathological bone resorption, we examined the effects of the FICD on bone erosion in a murine $\mathrm{K} / \mathrm{BxN}$ serum-transfer-induced arthritis mode ${ }^{30}$. K/BxN serum was administered intraperitoneally on days 0 and 2, and arthritis severity was assessed by clinical scores and ankle joint thickness until day 14. FICDtg ${ }^{\mathrm{M}}$ mice exhibited minimal differences in joint swelling or inflammation compared with littermate control mice in $\mathrm{K} / \mathrm{BxN}$ serum-induced arthritis (Fig. 5e, f). However, histomorphometric analysis revealed that osteoclast numbers, osteoclast surface areas, and the eroded surface in periarticular bone of FICDtg ${ }^{\mathrm{M}}$ mice were significantly increased compared to those of WT mice (Fig. $5 \mathrm{~g}, \mathrm{~h}$ ). Thus, our results suggest that the FICD promotes pathological bone loss under inflammatory conditions in vivo.

The FICD regulates RANKL-induced NFATc1 expression via the MNK1/2/elF4E axis

To gain insight into the mechanism by which FICD fragments regulate osteoclastogenesis, we examined the effect of the FICD on the expression of NFATc1, a master regulator of osteoclastogenesis ${ }^{31}$. Nfatc1 mRNA expression was comparable between WT and FICDtg ${ }^{\mathrm{M}}$ mice (Fig. 6a). However, RANKL-induced NFATc1 protein levels were substantially increased in FICDtg $^{\mathrm{M}}$ cells compared with WT cells (Fig. 6b). Consistently, NFATc1 protein expression was diminished by impaired FICD generation in FMS ${ }^{\text {mut }}$ cells compared with FMS ${ }^{\text {wt }}$ cells, while Nfatc1 mRNA expression was comparable between FMS ${ }^{\text {mut }}$ cells and FMS ${ }^{\text {wt }}$ cells (Fig. 6c, d). To explain the considerable discrepancy in Nfatc1 mRNA and protein levels between WT and FICDtg ${ }^{\mathrm{M}}$ osteoclasts, we examined 
a

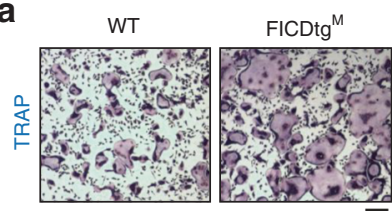

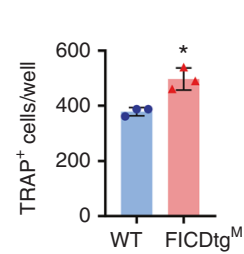

b WT

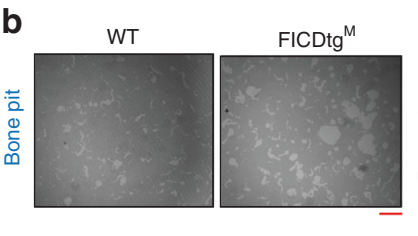

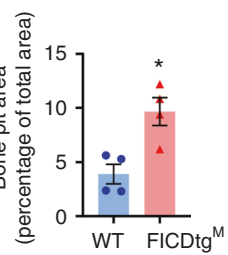

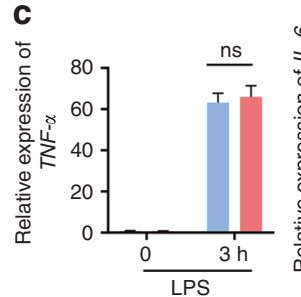

d

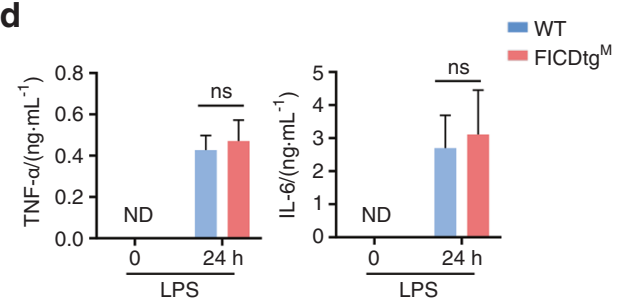

e

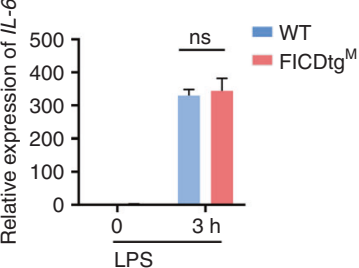

f

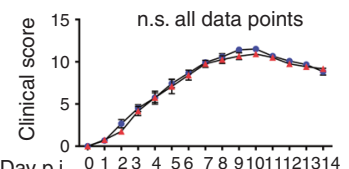

Dayp.i. 01234567891011121314

$$
\begin{aligned}
& \rightarrow \text { WT } \\
& \rightarrow \text { FICDtg}^{\mathrm{M}}
\end{aligned}
$$

g

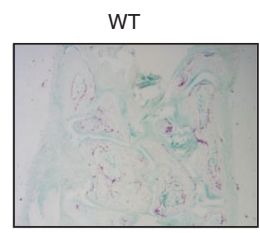

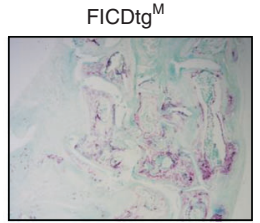

h

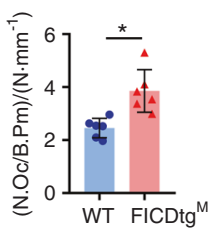

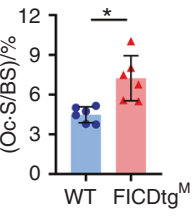

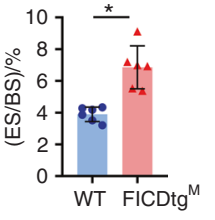

Fig. 5 FICDtg ${ }^{\mathrm{M}}$ mice show enhanced arthritic bone erosion. $\mathbf{a}, \mathbf{b}$ BMDMs from WT and FICDtg ${ }^{\mathrm{M}}$ mice were cultured with M-CSF and RANKL for 3 days. a Osteoclastogenesis assay. The left panel shows representative images of TRAP-stained cells. The right panel shows the percentage of TRAP-positive multinuclear cells per WT cell $(n=3)$. b Resorption pit assay. The left panel shows representative images, and the right panel shows the percentage of the resorbed pit area per total area $(n=3)$. BMDMs from WT and FICDtg ${ }^{\mathrm{M}}$ mice were stimulated with LPS $\left(10 \mathrm{ng} \cdot \mathrm{mL}^{-1}\right)$ for $3 \mathrm{~h}$ (c) and $24 \mathrm{~h}$ (d). c mRNA expression of TNF- $a$ and IL-6 was measured by q-PCR. d TNF $\alpha$ and IL6 protein levels in the culture media were measured by Luminex multiplex cytokine assays. $\mathbf{e}-\mathbf{h} \mathrm{K} / \mathrm{BxN}$ serum transfer-induced arthritis model. Eight-week-old female wildtype and FICDtg ${ }^{\mathrm{M}}$ mice were administered K/BxN serum on days 0 and 2. e, f Time course of joint swelling and clinical score analysis of seruminduced arthritis in littermate control and FICDtg ${ }^{\mathrm{M}}$ mice $(n=6)$. (g) Representative images of the TRAP-stained tarsal bones (hind paws) of arthritic mice. $\mathbf{h}$ Histomorphometric analysis of tarsal bones. N.Oc/B.Pm Osteoclast number/bone parameter, Oc.S/BS osteoclast surface/bone surface, ES/BS eroded surface/bone surface. The black scale bar is $100 \mu \mathrm{m}$, and the red scale bar is $200 \mu \mathrm{m}$. All data are shown as the mean \pm SEM. n.s. not significant. ND not detected. ${ }^{*} P<0.05$ two-way ANOVA with a post hoc Tukey test (c-f) or two-tailed, unpaired $t$-test (a, b, h).

the effect of the FICD on the activation of the mammalian target of the rapamycin (mTOR) pathway and on the induction of the MAPK interacting kinase (MNK1/2)-dependent pathway among several key signaling pathways that regulate protein translation ${ }^{32}$. We measured phospho-eIF4E as a downstream readout for the activation of the mTORC1 and MNK1/2 pathways. Strikingly, elF4E phosphorylation was activated by RANKL stimulation and was significantly increased in FICDtg ${ }^{\mathrm{M}}$ cells compared with WT cells (Fig. 6e), suggesting that the FICD may enhance elF4E-dependent protein synthesis. To further delineate the cause of the increased elF4E phosphorylation, we measured phospho-S6K and phospho4EBP1 to determine the activation of the mTORC1 pathway. RANKL-induced mTORC1 activation was comparable between FICDtg $^{\mathrm{M}}$ and control cells (Supplementary Fig. 10a, b). Consistent with the literature ${ }^{33}$, NFATc1 protein expression was comparable between control cells and RAPTOR-deficient cells, which are a model for low mTORC1 signaling (Supplementary Fig. 10c). Our data suggest that the MTORC1 pathway does not regulate FICDinduced elF4E phosphorylation. We next examined whether the MNK1/2-elF4E axis regulates RANKL-induced NFATc1 expression using the MNK1/2 inhibitor CGP57380 34 . Inhibiting MNK1/2 activity indeed suppressed RANKL-induced NFATc1 protein expression in a dose-dependent manner in both mouse and human macrophages, whereas Nfatc1 mRNA expression was marginally altered by CGP57380 treatment (Fig. 6f, g, Supplementary Fig. 10d, e). CGP57380 also suppressed osteoclast differentiation in a dose-dependent manner in $\mathrm{CD} 14^{+}$cells (Supplementary Fig. 10f). To examine the contribution of the MNK1/2 pathway to the increased osteoclastogenesis in $\mathrm{FICDtg}^{\mathrm{M}}$ cells, we treated
FICDtg $^{\mathrm{M}}$ cells with CGP57380. As expected, suppressing MNK1/2/ p-elF4E inhibited the enhanced osteoclastogenesis in FICDtg ${ }^{M}$ cells to levels comparable to those of WT osteoclasts (Fig. 6h). However, CGP57380 treatment induced minimal effects on cell viability (Fig. 6i). We examined the effect of CGP57380 on bone erosion in $\mathrm{K} / \mathrm{BxN}$ serum transfer-induced arthritis. K/BxN serum was administered intraperitoneally on days 0 and 2 , and then CGP57380 was administered after disease onset (Fig. 6j). Although the severity of arthritis was assessed by the clinical score and ankle joint thickness, and these factors were not affected by CGP57380 treatment (Fig. 6k, I), treatment with an MNK1/2 inhibitor decreased the number of osteoclasts and attenuated bone erosion in the K/BXN serum-induced arthritis model (Fig. $6 \mathrm{~m}$, n). Overall, our results suggest that increased phospho-elF4E is a key regulator of increased osteoclastogenesis in FICDtg ${ }^{\mathrm{M}}$ cells and that targeting the FICD/MNK1/2 axis significantly diminishes arthritic bone erosion.

FICD/DAP5/Fxr1 complexes activate the MNK1/2 pathway and NFATc1 expression

Next, we sought to identify the underlying mechanisms by which FICD increases elF4E phosphorylation. We performed an unbiased proteomic analysis using mass spectrometry with two biological replicates to screen proteins that both interact with the FICD and regulate the MNK1/2 pathway. FICD-DDK was transfected into 293 T cells, and FICD-interacting proteins were immunoprecipitated using anti-c-FMS antibodies. A total of 145 FICD-interacting proteins were identified (Supplemental Table 1). Ingenuity pathway analysis showed that 20 FICD-interacting proteins were 
a

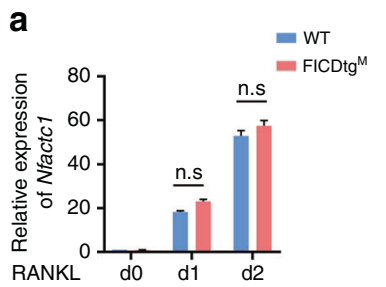

b

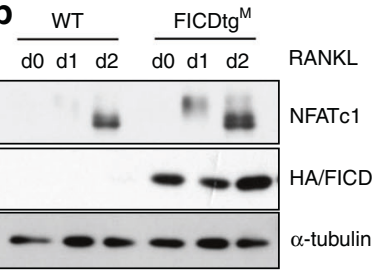

C

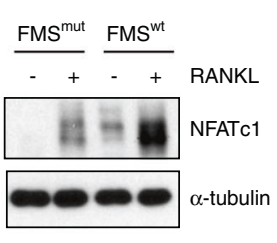

d

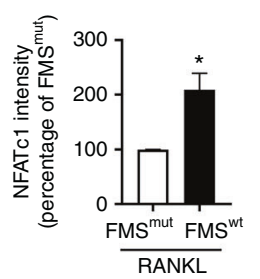

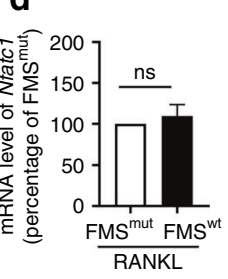

e

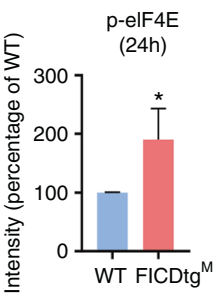

f
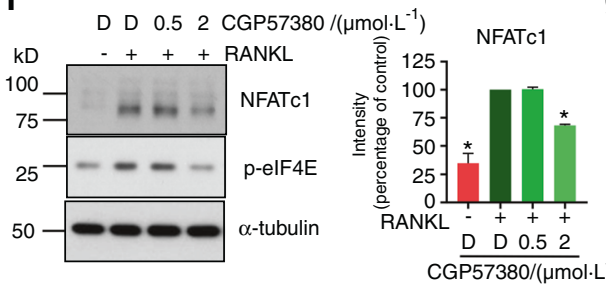

g

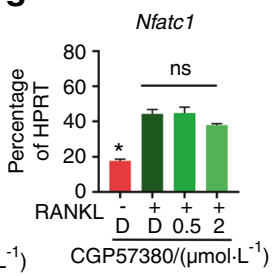

k
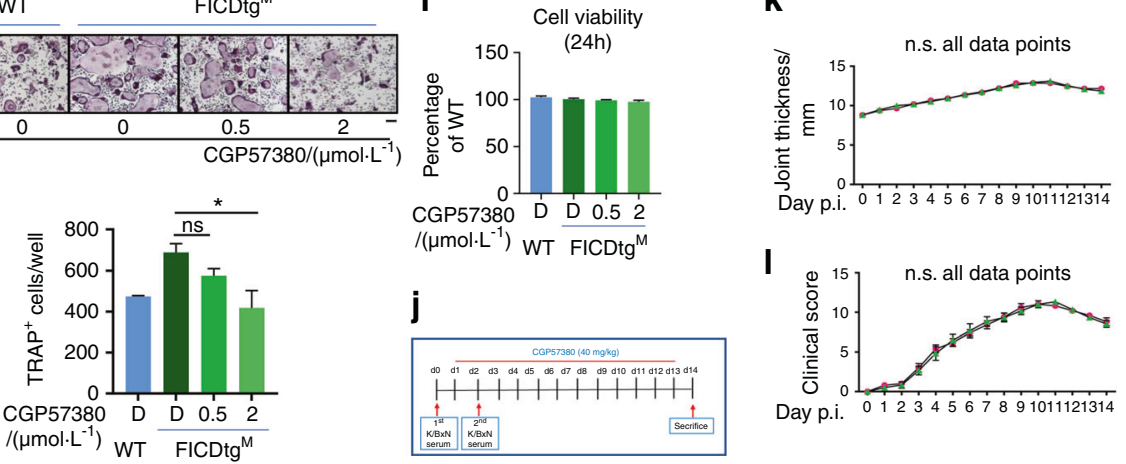

I

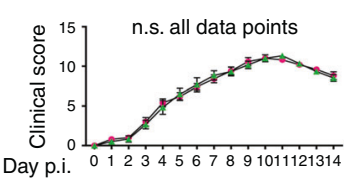

m

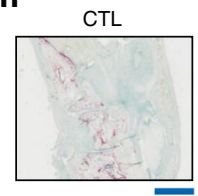

n

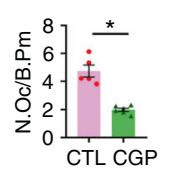

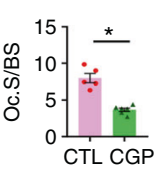

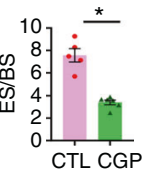

Fig. 6 The FICD augments NFATc1 expression by activating the MNK1/2/elF4E axis. a, b BMDMs from WT and FICDtg ${ }^{\mathrm{M}}$ mice were stimulated with RANKL $\left(50 \mathrm{ng} \cdot \mathrm{mL}^{-} 1\right.$ ) for the indicated times. a RT-qPCR analysis of Nfatc 1 mRNA normalized to Hprt mRNA. b Immunoblot analysis with anti NFATc1, HA, or $\alpha$-tubulin antibodies. c, d BMDMs from c-FMS ${ }^{\mathrm{f} /+} \mathrm{Mx} 1$-Cre mice were transduced with lentiviral particles encoding FMS ${ }^{\mathrm{wt}}$ or FMS $^{\text {mut }}$ and then cultured with M-CSF and RANKL. c Immunoblot analysis of whole cell lysate with anti-NFATc1 antibody. $\alpha$-Tubulin was used as a control. The left panel shows representative images. The right panel shows the cumulative intensity of NFATc1 bands. The intensity of NFATc1 in FMS ${ }^{\text {mut }}$ was set as $100 \%$. d The mRNA expression level of NFATc1. e Immunoblot analysis of whole cell lysates with phospho-elF4E antibodies. HA-tagged FICD was detected by HA-antibodies. $\alpha$-Tubulin was used as a control. The left panel shows representative images. The right panel shows the cumulative percentage of the intensity of the band (at $24 \mathrm{~h}$ ) relative to the control from three independent experiments. f, g BMDMs from WT mice were treated with CPG57380 at the indicated doses and then cultured with RANKL for 1 day. D: DMSO. f Immunoblot analysis with anti-NFATc1, phospho-eIF4E, or $\alpha$-tubulin antibodies. The left panel shows representative images. The right panel shows the cumulative percentage of the intensity of the band relative to the control (RANKL + DMSO) $(n=4)$. $\mathbf{g}$ Nfatc1 mRNA expression was measured by qPCR relative to Hprt mRNA. the DMSO-treated RANKL condition was set as $100 \%$. h Osteoclastogenesis assay. BMDMs from WT and FICDtg ${ }^{M}$ mice were treated with CPG57380 at the indicated doses and then cultured with RANKL for an additional 3 days. The upper panel shows representative images of TRAP-stained cells. The bottom panel shows the percentages of TRAP-positive multinuclear cells (MNCs: more than three nuclei) per control from three independent experiments. Scale bar: $100 \mu \mathrm{m}$. $\mathbf{i}$ Cell viability assay. BMDMs from WT and FICDtg $^{\mathrm{M}}$ mice were stimulated with CPG57380 at the indicated doses for 1 day. j-n K/BxN serum transfer-induced arthritis model. Nine-weekold male C57BL/6 J mice received K/BxN serum on days 0 and 2 . Vehicle or CPG57380 (40 mg.kg $\left.{ }^{-1}, C P G\right)$ was administered intraperitoneally (i. p.) from day 2 until day 13. j Schematic diagram showing the experimental design. k Ankle thickness. I Arthritis score. $\mathbf{m}$ Representative images of TRAP-stained histological sections from the calcaneocuboid and tarsometatarsal joints. Scale bar: $1 \mathrm{~mm}$. $\mathbf{n}$ Histomorphometric analysis of tarsal bones. N.OC/B.Pm Osteoclast number/bone parameter. OC.S/BS osteoclast surface/bone surface. ES/BS Eroded surface/bone surface. All data are shown as the mean \pm SEM. CTL, Control. ${ }^{*} P<0.05 ;$ n.s. not significant by one-way ANOVA with a post hoc Tukey test $(\mathbf{a}, \mathbf{f}-\mathbf{i}$, $\mathbf{k}$, I) or two-tailed, unpaired $t$-test $(\mathbf{c}, \mathbf{d}, \mathbf{e}, \mathbf{n})$. The data represent at least three experiments.

enriched in protein synthesis and posttranscriptional modifications (Fig. 7a). Among them, we focused on DAP5, which binds to MNK1 and is a part of protein translation initiation complexes ${ }^{35}$ (Fig. 7b, c). To verify the interaction between the FICD and DAP5, we performed immunoprecipitation analysis using BMDMs from wild-type and FICDtg ${ }^{M}$ mice. We found that the FICD bound to DAP5 (Fig. 7d). As Fxr1 was shown to form a complex with DAP5 ${ }^{36}$, we also examined whether Fxr1 interacted with the FICD. The FICD also bound to Fxr1 (Fig. 7d), suggesting that the FICD might interact with the DAP5/Fxr1 complex.

As the function of the DAP5/Fxr1 complex in osteoclasts has not been previously characterized, we examined the effect of the DAP5/Fxr1 complex on osteoclastogenesis by knocking down these proteins in both human and murine osteoclasts using siRNAs. Both DAP5 and Fxr1 were increased upon RANKL stimulation, and knockdown of DAP5 and Fxr1 suppressed their expression in both human and murine macrophages (Supplementary Fig. 10g-j). Strikingly, DAP5 or Fxr1 deficiency suppressed RANKL-induced elF4E phosphorylation and NFATC1 protein expression (Fig. 7e-h), suggesting that the FICD/DAP5/Fxr1 axis plays an important role in elF4E phosphorylation and NFATc1 expression in osteoclasts. Accordingly, osteoclast differentiation was also suppressed by DAP5 or Fxr1 deficiency (Fig. 7i, j). Our data suggest that the DAP5/Fxr1 complex contributes to the activation of MNK1/2/elF4E and NFATc1 expression in osteoclasts and serves as a positive regulator of osteoclast differentiation. 


\begin{tabular}{|ccc|}
\hline Functions & $P$-value & $\begin{array}{c}\text { No. of } \\
\text { molecules }\end{array}$ \\
$\begin{array}{c}\text { Gene expression } \\
\text { (Expression of RNA) } \\
\text { Protein synthesis } \\
\text { (Translation of protein) } \\
\quad \text { Gene expression } \\
\text { (Stabilization of mRNA) }\end{array}$ & $1.88 \mathrm{E}-09$ & 69 \\
$\begin{array}{c}\text { DNA replications, } \\
\text { recombination, and repair } \\
\text { Protein synthesis } \\
\text { (Expression of protein) }\end{array}$ & $2.10 \mathrm{E}-07$ & 20 \\
\hline
\end{tabular}

b

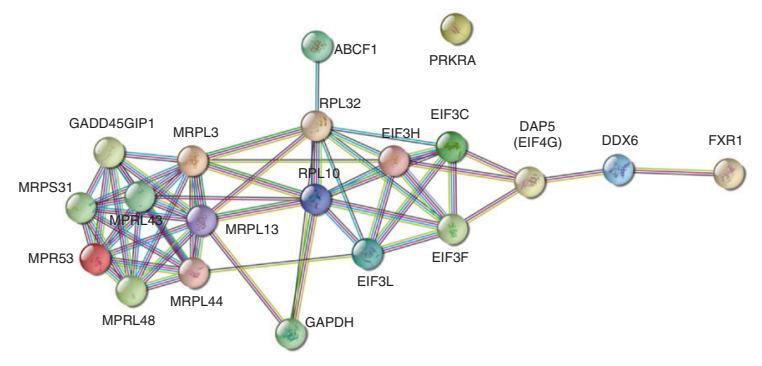

C FICD binding proteins enriched in "Protein synthesis pathways"

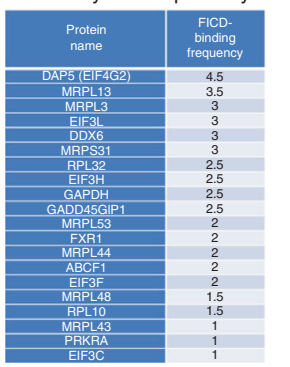

d

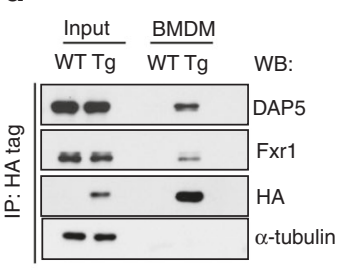

e

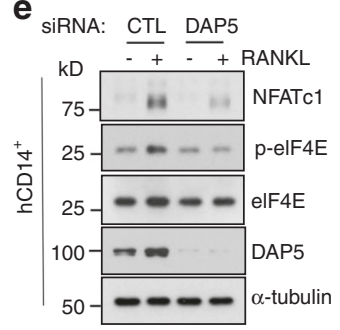

f

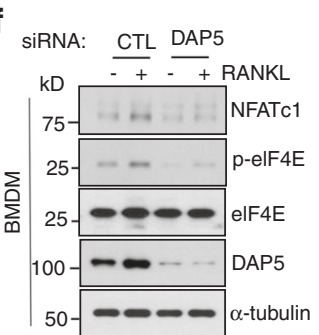

$\mathbf{g}_{\text {siRNA: CTL Fxr1 }}$

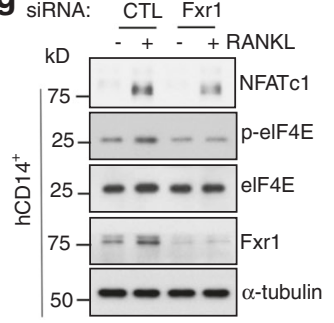

h


Fig. 7 The FICD enhances the activation of MNK1/2/elF4E via DAP5/Fxr1. a Ingenuity pathway analysis of 145 FICD-interacting proteins. Pooled data from two biological replicates were analyzed. b Interaction map showing 20 FICD-interacting proteins in "Protein synthesis pathways" using STRING functional protein association analysis. c Frequencies of the proteins shown in (b). d The interaction of the FICD with DAP5 or Fxr1 was determined by immunoblot analysis with anti-DAP5, Fxr1, HA, or $\alpha$-tubulin antibodies. Whole cell lysates of BMDMs from WT and FICDtg ${ }^{\mathrm{M}}$ mice were used for immunoprecipitation with anti-HA-tagged antibodies. Knockdown (KD) of DAP 5 (e, $\left.\mathbf{f}, \mathbf{i}, \mathbf{j}\right)$ or Fxr1 (g, h, i, j) in both human $\mathrm{CD}_{14}^{+}$cells $(\mathbf{e}, \mathbf{g}, \mathbf{i})$ and BMDMs $(\mathbf{f}, \mathbf{h}, \mathbf{j})$. e-h The protein expression of NFATc1, p-elF4E, elF4E, DAP5, Fxr1, and $\alpha$-tubulin was determined by immunoblot analysis. Osteoclastogenesis assay. KD of DAP5 or Fxr1 in hCD14 ${ }^{+}$cells (i) or in BMDMs (j) that were cultured with M-CSF and RANKL for 3 days. The left panel shows representative images of TRAP-stained cells. The right panel shows the percentages of TRAP-positive multinuclear cells (MNCs: more than three nuclei) per control from three independent experiments. CTL Control siRNAs. All data are shown as the mean \pm SEM. ${ }^{*} P<0.05$ by one-way ANOVA with a post hoc Tukey test (i, $\left.\mathbf{j}\right)$. The data represent 2 biological replicates for mass spectrometry $(\mathbf{a}-\mathbf{c})$ and three independent experiments $(\mathbf{d}-\mathbf{i})$.

Taken together, our findings suggest that the FICD promotes osteoclast differentiation by permitting the sustained activation of MNK1/2 and eIF4E phosphorylation, and in turn, NFATc1 expression is increased and enhances FICDtg $^{\mathrm{M}}$ osteoclast formation (Supplementary Fig. 11).

\section{DISCUSSION}

Cell surface receptors sense environmental stimuli and control cellular responses by activating downstream signaling cascades. However, recent studies have revealed that intramembrane cleavage of cell surface receptors also plays an important role in signaling processes and regulates cellular functions. Here, we demonstrated that c-FMS proteolysis was critically involved in the osteoclastogenic responses of macrophages to RANKL and cooperated with the conventional M-CSF/c-FMS signaling pathways. c-FMS is processed into smaller intracellular fragments (FICD fragments) in macrophages by engaging c-FMS-mediated signaling pathways. FICD fragments form a complex with DAP5 and activate the MNK1/2-elF4E axis to enhance NFATC1 protein expression and osteoclastogenesis. Our data established the FICD as a positive regulator of osteoclastogenesis. Furthermore, by modeling the increase in FICD fragments in RA macrophages, myeloid cell-specific FICD expression enhanced in vivo osteoclastogenesis and promoted arthritic bone erosion in a murine arthritis model. These findings identify a novel function of c-FMS proteolysis in regulating (patho)physiological bone erosion and sensitivity to stimulation with the cytokine RANKL.

The altered expression of M-CSF and c-FMS has been implicated in the exacerbation of various diseases ${ }^{37}$. To readjust the c-FMS-MCSF/IL-34 axis, several drug discovery programs have been focused on finding inhibitors of the tyrosine kinase activity of $\mathrm{C}^{-\mathrm{FMS}^{38}}{ }^{38}$. Although inhibiting c-FMS kinase activity appears to be an attractive strategy and has already shown promise, the prolonged use of c-FMS inhibitors is limited by their side effects. Targeting osteoclasts using denosumab, an anti-RANKL antibody, shows efficacy on the progression of arthritic bone erosion without affecting RA disease activity ${ }^{39,40}$, emphasizing the importance of osteoclasts in arthritic bone erosion. A better understanding of osteoclast regulation in arthritis is important for developing osteoclast-specific therapeutic interventions for arthritic bone erosion. We demonstrated that FICD overexpression using transgenic FICD knock-in mice affected osteoclasts with no effect on disease activity, while inhibiting c-FMS signaling attenuated both disease activity and arthritic bone erosion in murine arthritis models. This finding was consistent with our observations that blocking c-FMS proteolysis had no effect on inflammation. In normal macrophages, the FICD level was very low. However, high FICD expression was found in RA synovial CD14 ${ }^{+}$cells, which have increased potential to differentiate into 
osteoclasts. Many plausible causes of arthritic bone erosion, including chronic inflammation, have been identified. In addition, predicative markers for arthritic bone erosion have been investigated. The correlation between serological markers and the radiographic progression in RA has been identified in a longterm longitudinal study, while some studies have shown a negative correlation between serological markers and bone destruction in $\mathrm{RA}^{41-44}$. We did not find a correlation between the levels of FICD fragments and serological markers in RA. Since our study had a small number of cohorts, further studies will be needed to determine whether the level of FICD fragments can be used as a predicative marker for arthritic bone erosion. Taken together, our findings revealed the pathophysiological importance of the FICD and its associated pathways in arthritic bone erosion and suggests that inhibiting FICD generation or function in RA patients who have high FICD expression in macrophages might be beneficial for inflammatory bone destruction.

Our results demonstrated that c-FMS proteolysis was involved not only in protein turnover but also in generating the necessary functional elements to promote osteoclastogenesis. High levels of $M-C S F$ in the RA synovium and RA synovial fluids may contribute to c-FMS proteolysis and FICD generation. It has been shown that c-FMS proteolysis generates small fragments via TACE and $\gamma^{-}$ secreatase $^{10}$. c-FMS proteolysis has been considered a disposal mechanism that is coupled with proteosomal degradation of c-FMS. Enhancing c-FMS degradation suppresses osteoclastogenesis $^{45-47}$. When macrophages are exposed to inflammatory mediators, c-FMS is rapidly degraded, and osteoclastogenesis is suppressed by depleting RANK ${ }^{45}$. Inhibiting proteosomal degradation with bortezomib promotes c-FMS degradation and suppresses osteoclastogenesis ${ }^{46,47}$. However, the role of small fragments in macrophages/osteoclasts remains unknown. We identified the function of FICD fragments in myeloid cells under physiological and pathological conditions. Moreover, our data showed that the accumulation of FICD fragments was associated with pathological conditions such as RA. We also showed that FMS $^{\text {mut }}$ did not generate FICD fragments and exhibited impaired osteoclastogenesis. Although we demonstrated that FMS ${ }^{\text {mut }}$ was a functional receptor, we could not exclude the potential effects of $\mathrm{FMS}^{\text {mut }}$ on other signaling pathways that play an important role in osteoclastogenesis. Thus, we used the FICD overexpression system in myeloid cells. Intriguingly, the effect of FICD fragments on the inflammatory response was negligible, suggesting the specific activity of the FICD on osteoclastogenesis. Our data from FICDtg ${ }^{\mathrm{M}}$ mice suggest that impaired FICD generation in FMS ${ }^{\text {mut }}$ is likely to affect osteoclastogenesis. Although we screened the candidate protease that cleaves c-FMS using a protease inhibitor library that includes 53 known protease inhibitors, it is still possible that other proteases are involved in c-FMS proteolysis. Our study extended the current paradigm of the c-FMS signaling network by demonstrating that c-FMS proteolysis is a new player in the c-FMS signaling network.

The MNK1/2/p-elF4E axis is a downstream mediator of FICD fragments and interacts with DAP5/Fxr 1 complexes. The role of DAP5 and Fxr1 in osteoclasts has not been explored. We showed that DAP5 or Fxr1 deficiency suppressed NFATc1 expression and osteoclastogenesis. However, FICD/DAP5/Fxr1 complexes can target other proteins than NFATC1 to suppress osteoclastogenesis. Further investigation of the mechanisms by which c-FMS proteolysis regulates the function of DAP5 is needed for a deeper understanding. Moreover, inhibiting MNK1/2 activity suppressed osteoclastogenesis and bone erosion in a K/BXN serum-transfer arthritis model. Thus, our study provides important insights into the amenability of the FICD/DAP5/Fxr1/MNK1/2 axis to therapeutic intervention. In addition, blocking the pathways involved in the generation of the FICD can be a potential therapeutic strategy for osteoclast-mediated diseases. Overall, the effects of the FICD on osteoclast differentiation and bone resorption under pathological conditions can be determined by the levels of $M$ CSF, the activation of proteases, and the availability of FICDinteracting proteins.

In summary, our findings highlight the importance of c-FMS proteolysis in c-FMS-mediated signaling pathways in macrophages/ osteoclasts and identify the mechanisms by which FICD generation and nuclear translocation occur. Our study also identifies a new pathway by which osteoclast differentiation and activity are enhanced in the pathogenesis of osteoclast-mediated bone diseases, which may be useful for developing innovative therapeutic interventions that specifically target osteoclast activity.

\section{METHODS}

Methods are available in the Supplementary material.

\section{ACKNOWLEDGEMENTS}

We would like to thank Dr. Sungho Park for his helpful discussions and review of the manuscript, Drs. Miguel Otero and Lionel Ivashkiv for providing human patient samples and K/BXN serum and the HSS micro-CT core. This work was supported by the National Institute of Arthritis and Musculoskeletal and Skin Diseases (NIAMS) of the NIH under Award Numbers R01 AR069562 and AR073156 (to K.H.P.-M.). Supplementary Figure 11 was generated by Biorender.

\section{AUTHOR CONTRIBUTIONS}

S.M. conceived and designed the projects and wrote the manuscript. S.M., S.B., S.Z., B.O., C.C., M.J.K., H.K., and C.L.D. performed the experiments. G.K., Y.O., T.K., and J.J. performed human sample experiments and expertise. K.H.P.-M. conceived, designed and oversaw the project and edited the manuscript. All authors reviewed and provided input on the manuscript.

\section{ADDITIONAL INFORMATION}

Supplementary information The online version contains supplementary material available at https://doi.org/10.1038/s41413-021-00162-0.

Competing interests: The authors declare no competing interests.

\section{REFERENCES}

1. Smolen, J. S. et al. Rheumatoid arthritis. Nat. Rev. Dis. Prim. 4, 18001 (2018).

2. Schett, G. \& Gravallese, E. Bone erosion in rheumatoid arthritis: mechanisms, diagnosis and treatment. Nat. Rev. Rheumatol. 8, 656-664 (2012).

3. Guo, Q. et al. Rheumatoid arthritis: pathological mechanisms and modern pharmacologic therapies. Bone Res 6, 15 (2018).

4. Lin, H. et al. Discovery of a cytokine and its receptor by functional screening of the extracellular proteome. Science 320, 807-811 (2008).

5. Paniagua, R. T. et al. c-Fms-mediated differentiation and priming of monocyte lineage cells play a central role in autoimmune arthritis. Arthritis Res Ther. 12, R32 (2010).

6. Ohno, $\mathrm{H}$. et al. The orally-active and selective c-Fms tyrosine kinase inhibitor Ki20227 inhibits disease progression in a collagen-induced arthritis mouse model. Eur. J. Immunol. 38, 283-291 (2008).

7. Pollard, J. W. Trophic macrophages in development and disease. Nat. Rev. Immunol. 9, 259-270 (2009).

8. Kühnle, N., Dederer, V. \& Lemberg, M. K. Intramembrane proteolysis at a glance: from signalling to protein degradation. J. Cell Sci. 132, jcs 217745 (2019).

9. Ivashkiv, L. B., Zhao, B., Park-Min, K. H. \& Takami, M. Feedback inhibition of osteoclastogenesis during inflammation by IL-10, M-CSF receptor shedding, and induction of IRF8. Ann. N. Y Acad. Sci. 1237, 88-94 (2011).

10. Vahidi, A., Glenn, G. \& van der Geer, P. Identification and mutagenesis of the TACE and gamma-secretase cleavage sites in the colony-stimulating factor 1 receptor. Biochem. Biophys. Res. Commun. 450, 782-787 (2014).

11. Glenn, G. \& van der Geer, P. CSF-1 and TPA stimulate independent pathways leading to lysosomal degradation or regulated intramembrane proteolysis of the CSF-1 receptor. FEBS Lett. 581, 5377-5381 (2007).

12. Tsukasaki, M. \& Takayanagi, H. Osteoimmunology: evolving concepts in boneimmune interactions in health and disease. Nat. Rev. Immunol. 19, 626-642 (2019). 
13. Park-Min, K. H. Mechanisms involved in normal and pathological osteoclastogenesis. Cell Mol. Life Sci. 75, 2519-2528 (2018).

14. Novack, D. V. \& Teitelbaum, S. L. The osteoclast: friend or foe? Annu Rev. Pathol. 3 457-484 (2008).

15. Hamilton, J. A. Colony-stimulating factors in inflammation and autoimmunity. Nat. Rev. Immunol. 8, 533-544 (2008).

16. Ross, F. P. \& Teitelbaum, S. L. Alphavbeta3 and macrophage colony-stimulating factor: partners in osteoclast biology. Immunol. Rev. 208, 88-105 (2005).

17. Gebauer, F. \& Hentze, M. W. Molecular mechanisms of translational control. Nat. Rev. Mol. Cell Biol. 5, 827-835 (2004).

18. Imataka, H., Olsen, H. S. \& Sonenberg, N. A new translational regulator with homology to eukaryotic translation initiation factor $4 G$. EMBO J. 16, 817-825 (1997).

19. Yoffe, Y. et al. Cap-independent translation by DAP5 controls cell fate decisions in human embryonic stem cells. Genes Dev. 30, 1991-2004 (2016).

20. Park-Min, K. H. et al. Inhibition of osteoclastogenesis and inflammatory bone resorption by targeting BET proteins and epigenetic regulation. Nat. Commun. 5, 5418 (2014).

21. Lanz, T. A. et al. The gamma-secretase inhibitor N-[N-(3,5-difluorophenacetyl)-Lalanyl]-S-phenylglycine t-butyl ester reduces $A$ beta levels in vivo in plasmaand cerebrospinal fluid in young (plaque-free) and aged (plaque-bearing) Tg2576 mice. J. Pharmacol. Exp. Ther. 305, 864-871 (2003).

22. Stanley, E. R. \& Chitu, V. CSF-1 receptor signaling in myeloid cells. Cold Spring Harb. Perspect. Biol. 6, a021857 (2014).

23. Pfaff, M., Du, X. \& Ginsberg, M. H. Calpain cleavage of integrin beta cytoplasmic domains. FEBS Lett. 460, 17-22 (1999).

24. Deshpande, R. V. et al. Calpain expression in lymphoid cells. Increased mRNA and protein levels after cell activation. J. Biol. Chem. 270, 2497-2505 (1995).

25. Svensson, L. et al. Calpain 2 controls turnover of LFA-1 adhesions on migrating $T$ lymphocytes. PLoS One 5, e15090 (2010).

26. Marzia, M. et al. Calpain is required for normal osteoclast function and is downregulated by calcitonin. J. Biol. Chem. 281, 9745-9754 (2006).

27. Yaroslavskiy, B. B., Sharrow, A. C., Wells, A., Robinson, L. J. \& Blair, H. C. Necessity of inositol $(1,4,5)$-trisphosphate receptor 1 and mu-calpain in NO-induced osteoclast motility. J. Cell Sci. 120, 2884-2894 (2007).

28. Kuhn, R., Schwenk, F., Aguet, M. \& Rajewsky, K. Inducible gene targeting in mice. Science 269, 1427-1429 (1995).

29. Liu, Z. et al. GPS-CCD: a novel computational program for the prediction of calpain cleavage sites. PLoS One 6, e19001 (2011).

30. Kouskoff, V. et al. Organ-specific disease provoked by systemic autoimmunity. Cell 87, 811-822 (1996).

31. Negishi-Koga, T. \& Takayanagi, H. Ca ${ }^{2+}$-NFATc1 signaling is an essential axis of osteoclast differentiation. Immunol. Rev. 231, 241-256 (2009).

32. Sonenberg, N. \& Hinnebusch, A. G. Regulation of translation initiation in eukaryotes: mechanisms and biological targets. Cell 136, 731-745 (2009).

33. Huynh, H. \& Wan, Y. mTORC1 impedes osteoclast differentiation via calcineurin and NFATc1. Commun. Biol. 1, 29 (2018).

34. Grzmil, M. et al. MNK1 pathway activity maintains protein synthesis in rapalogtreated gliomas. J. Clin. Investig. 124, 742-754 (2014).
35. Pyronnet, S. et al. Human eukaryotic translation initiation factor $4 \mathrm{G}$ (elF4G) recruits mnk1 to phosphorylate elF4E. EMBO J. 18, 270-279 (1999).

36. Bukhari, S. I. A. et al. A specialized mechanism of translation mediated by FXR1aassociated microRNP in cellular quiescence. Mol. Cell 61, 760-773 (2016).

37. Mun, S. H., Park, P. S. U. \& Park-Min, K. H. The M-CSF receptor in osteoclasts and beyond. Exp. Mol. Med. 52, 1239-1254 (2020).

38. Hamilton, J. A., Cook, A. D. \& Tak, P. P. Anti-colony-stimulating factor therapies for inflammatory and autoimmune diseases. Nat. Rev. Drug Discov. 16, 53-70 (2016).

39. Ishiguro, N. et al. Efficacy of denosumab with regard to bone destruction in prognostic subgroups of Japanese rheumatoid arthritis patients from the phase II DRIVE study. Rheumatol. (Oxf.) 58, 997-1005 (2019).

40. Cohen, S. B. et al. Denosumab treatment effects on structural damage, bone mineral density, and bone turnover in rheumatoid arthritis: a twelve-month, multicenter, randomized, double-blind, placebo-controlled, phase II clinical trial. Arthritis Rheum. 58, 1299-1309 (2008).

41. Syversen, S. W. et al. High anti-cyclic citrullinated peptide levels and an algorithm of four variables predict radiographic progression in patients with rheumatoid arthritis: results from a 10-year longitudinal study. Ann. Rheum. Dis. 67, 212-217 (2008).

42. Lindqvist, E., Eberhardt, K., Bendtzen, K., Heinegard, D. \& Saxne, T. Prognostic laboratory markers of joint damage in rheumatoid arthritis. Ann. Rheum. Dis. 64, 196-201 (2005).

43. Kim, K. W., Kim, B. M., Moon, H. W., Lee, S. H. \& Kim, H. R. Role of C-reactive protein in osteoclastogenesis in rheumatoid arthritis. Arthritis Res. Ther. 17, 41 (2015).

44. Cho, I. J. et al. Effects of C-reactive protein on bone cells. Life Sci. 145, 1-8 (2016).

45. Carlberg, K., Tapley, P., Haystead, C. \& Rohrschneider, L. The role of kinase activity and the kinase insert region in ligand-induced internalization and degradation of the c-fms protein. EMBO J. 10, 877-883 (1991).

46. Terpos, E., Sezer, O., Croucher, P. \& Dimopoulos, M. A. Myeloma bone disease and proteasome inhibition therapies. Blood 110, 1098-1104 (2007).

47. Lee, K. et al. Blocking of the ubiquitin-proteasome system prevents inflammationinduced bone loss by accelerating M-CSF receptor c-Fms degradation in osteoclast differentiation. Int. J. Mol. Sci. 18, 2054 (2017).

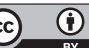

Open Access This article is licensed under a Creative Commons Attribution 4.0 International License, which permits use, sharing, adaptation, distribution and reproduction in any medium or format, as long as you give appropriate credit to the original author(s) and the source, provide a link to the Creative Commons license, and indicate if changes were made. The images or other third party material in this article are included in the article's Creative Commons license, unless indicated otherwise in a credit line to the material. If material is not included in the article's Creative Commons license and your intended use is not permitted by statutory regulation or exceeds the permitted use, you will need to obtain permission directly from the copyright holder. To view a copy of this license, visit http://creativecommons. org/licenses/by/4.0/.

(c) The Author(s) 2021 